\title{
Ex vivo culture of intestinal crypt organoids as a model system for assessing cell death induction in intestinal epithelial cells and enteropathy
}

\author{
T Grabinger ${ }^{1}$, L Luks ${ }^{1}$, F Kostadinova ${ }^{1}$, C Zimberlin², JP Medema ${ }^{2}$, M Leist ${ }^{3}$ and T Brunner ${ }^{\star, 1}$
}

Intestinal epithelial cells (IECs) not only have a critical function in the absorption of nutrients, but also act as a physical barrier between our body and the outside world. Damage and death of the epithelial cells lead to the breakdown of this barrier function and inflammation due to access of the immune system to compounds of the intestinal flora. Intestinal epithelial damage is frequently associated with various inflammatory disorders, chemo- and radiotherapy as well as drug-mediated toxicity. Until recently, intestinal epithelial-damaging activities of drugs and treatments could be tested only in vivo in animal models because of the poor survival rate of primary IECs ex vivo. The three-dimensional culture and outgrowth of intestinal crypt stem cells into organoids have offered new possibilities to culture and study IECs ex vivo. Here we demonstrate that intestinal organoids are a useful and physiologically relevant model system to study cell death and survival in IECs. We further describe a number of microscopy-based as well as colorimetric methods to monitor and score survival and death of intestinal organoids. Finally, the comparison of organoids isolated from gene-deficient mice and wild-type mice allows investigating the role of specific genes in the regulation of IEC death. Owing to their comparable structure and behavior, intestinal organoids may serve as an interesting and physiologically relevant surrogate system for large- and mid-scale in vitro testing of intestinal epithelium-damaging drugs and toxins, and for the investigation of cell death pathways.

Cell Death and Disease (2014) 5, e1228; doi:10.1038/cddis.2014.183; published online 15 May 2014

Subject Category: Experimental Medicine

The intestinal epithelium is an incredibly large mucosal surface with an extreme self-renewing capacity. ${ }^{1,2}$ Extensive proliferation of progenitor cells in the intestinal crypts and their differentiation into mature epithelial cells continuously compensates for cell loss at the villus tip by anoikis. However, in various pathological disorders or after medical treatments, the homeostasis between proliferation and cell death can become substantially disturbed, resulting in loss of epithelial integrity and local inflammation because of the increased stimulation of the immune system by the products of the intestinal flora. ${ }^{3}$ For instance in Crohn's disease ${ }^{4}$ and ulcerative colitis, ${ }^{5}$ coeliac disease, ${ }^{6}$ graft-versus-host disease ${ }^{7}$ and sepsis, ${ }^{8}$ the uncontrolled activation of the immune system results in the development of cytotoxic effector functions, and release of cytokines, which either directly damage the intestinal epithelium by inducing apoptosis or disrupt the epithelial tight junctions in between the epithelial cells. ${ }^{9}$ The subsequent invasion of intestinal bacteria and the associated stimulation of the immune system further accelerate the course of the disease ${ }^{10}$ In particular members of the tumor necrosis factor (TNF) superfamily, such as, TNF $\alpha$ and Fas (CD95) ligand have been shown to mediate immune cell-mediated damage of the intestinal epithelium. ${ }^{11-13}$ Furthermore, TNF $\alpha$ and IFN $\gamma$ contribute to epithelial barrier damage by causing disruption of tight junctions. ${ }^{14}$

Next to immune cell-mediated damage, various toxins and pharmacological drugs are known to cause unspecific and excessive cell death in the intestinal epithelium. ${ }^{15-18}$ Owing to its high-proliferative potential, the intestinal epithelium is also target of DNA damage and cell death associated with chemotherapy and radiation therapy. ${ }^{19-21}$ Not surprisingly, enteropathy and associated diseases are a frequent adverse side-effect of therapeutic treatments of tumor patients.

To distinguish between the positive therapeutic effects of pharmacological drugs and their potentially adverse sideeffects is a major goal of preclinical and clinical trials. Ideally, adverse or even toxic effects on primary cells are seen early enough in preclinical studies to avoid unnecessary in vivo testings in animals or to prevent harmful exposure of human patients. Cellular in vitro models may represent interesting tools to predict the potential toxic effects of drugs and treatments to be tested. Preferentially, in vitro testing of given drugs should be done in primary intestinal epithelial cells (IECs). However, isolated IECs rapidly die when cultured

\footnotetext{
${ }^{1}$ Chair of Biochemical Pharmacology, Department of Biology, University of Konstanz, Konstanz, Germany; ${ }^{2}$ Laboratory for Experimental Oncology and Radiobiology, Center for Experimental Molecular Medicine, Academic Medical Center, University of Amsterdam, Amsterdam, The Netherlands and ${ }^{3}$ Doerenkamp-Zbinden Chair of In-Vitro Toxicology and Biomedicine, Department of Biology, University of Konstanz, Konstanz, Germany

${ }^{*}$ Corresponding author: T Brunner, Chair of Biochemical Pharmacology, Department of Biology, University of Konstanz, Universitätsstrasse 10, Konstanz 78467, Germany. Tel: + 49753188 5371; Fax: + 49753188 5372; E-mail: thomas.brunner@uni-konstanz.de

Keywords: intestinal crypts; apoptosis; tissue damage; enteropathy; side-effects; drug screening

Abbreviations: 5-FU, 5-fluorouracyl; ActD, actinomycin D; IEC, intestinal epithelial cells; MTT, 3-(4,5-dimethylthiazol-2-yl)-2,5-diphenyltetrazolium bromide; $\mathrm{PI}$, propidium iodide; TNF, tumor necrosis factor

Received 16.1.14; revised 26.3.14; accepted 27.3.14; Edited by M Leverkus
} 
ex vivo. ${ }^{22}$ The cell death of isolated IECs is so rapid that an accelerated death promoted by a drug or treatment can often not be observed. Therefore, IEC-derived immortalized cell lines or tumor cells are frequently used as surrogates; however, immortalization or transformation often alters the intestinal epithelial-specific response to drugs and treatments. In particular, intestinal tumor cells have been selected to survive harsh environments, and are frequently characterized by high expression levels of anti-apoptotic gene products, such as members of the Bcl-2 family. ${ }^{23}$ Consequently, this may result in increased resistance to pharmacological drugs or irradiation, unrepresentative for the sensitivity of primary epithelial cells. Thus, data obtained with cell lines have to be interpreted carefully and do often not allow to draw conclusions on the in vivo situation in the intestinal mucosa. In vivo testing in mice represents a more physiological alternative. Such in vivo experiments are, however, cost intensive and generate often only limited data end points as mice have to be killed to assess damage in the intestinal epithelium using histology or histochemistry. Furthermore, toxicological studies in animals are also largely limited by ethical considerations.

Recently, Sato et al. presented a novel method that allows long-term culture of isolated intestinal crypts or intestinal stem cells. ${ }^{24}$ Supplemented with the appropriate growth factor cocktail (epidermal growth factor, Noggin, R-spondin-1) and cultured in a three-dimensional extracellular matrix, these intestinal stem cells are capable of developing into organoids and enteroids, displaying many important functions of the normal intestinal epithelium (mini-guts). This culture model has proven to serve as a powerful system to investigate regulatory and pathological mechanisms of the intestinal epithelium on a molecular level. ${ }^{25}$ Of note, the supplemented growth factors in the culture medium are identical with the signals that regulate intestinal stem cell niches in vivo. ${ }^{26}$ Thus far, this system has been extensively used for studying the regulation of intestinal stem cell self-renewal, growth and differentiation. In addition, in vitro expanded organoids may be used for gastrointestinal stem cell therapy in preclinical animal models. ${ }^{27}$ More recent applications also involve studies on colorectal tumor stem cells. ${ }^{28}$

Here, we now show that intestinal organoids, grown out of primary intestinal crypts, are an interesting and suitable model to study toxicity and cell death induction in ex vivo cultured primary epithelial cells. The model system is easy to use and allows quantification of cell death. We further show that cell death responses of organoids largely differ from that of colon carcinoma cells often used as surrogates. Last, intestinal organoids from gene-deficient mice allow to assess the impact of certain gene products on cell death induction in these primary epithelial cells. Thus, intestinal organoids may represent an ideal tool for preclinical toxicological studies on physiologically relevant primary IECs.

\section{Results}

Organoid growth and survival. When crypts isolated from small intestine were cultured in Matrigel, they closed and formed organoids ${ }^{29}$ - transparent sphere-like structures - within the first hours of culture (Supplementary Figure 1a). After
2-3 days, the spheres started to bud, and after 7 days, enteroids ${ }^{29}$ with numerous crypt-like structures were formed. When isolated crypts were cultured in medium lacking R-spondin-1, crypts readily closed to form organoids, but then stopped growing and appeared to die after 1 day of culture. Surviving and dying organoids were easily distinguished by the drastic change in organoid morphology with loss of epithelial integrity and impaired lumen formation (Supplementary Figure 1a). Subsequently, organoids with this dying phenotype were termed 'disrupted organoids'. Likewise, withdrawal of R-spondin-1 from fully grown enteroids resulted in the same morphologic changes, indicating that R-spondin-1 is not only required for organoid growth but also for enteroid survival (Supplementary Figure 1b).

Apoptosis triggers promote organoid disruption. To assess whether organoid disruption can be actively induced, we exposed day 3 organoids to various triggers known to promote IEC apoptosis. TNF $\alpha$ is a potent inducer of IEC apoptosis and thereby contributes to the pathogenesis of various inflammatory disorders of the intestine. ${ }^{30}$ When organoids were exposed to murine $\operatorname{TNF} \alpha$, a concentrationdependent increase in the number of organoids with disrupted morphology was observed (Figure 1a), suggesting that $\mathrm{TNF} \alpha$ was promoting organoid death. Quantification revealed that concentrations of TNF $\alpha$ as low as $10 \mathrm{ng} / \mathrm{ml}$ promoted a substantial increase in the number of crypts with a disrupted phenotype (Figure 1b). Next to TNF $\alpha$ as a representative biological cell death trigger, we investigated the response of organoids to cisplatin and UV irradiation. Both treatments also resulted in a significantly increased number of organoids with lost integrity, compared with untreated control organoids (Figure 1c). These findings confirm that treatment of organoids with archetypic cell death-inducing triggers results in an altered phenotypic appearance and the disintegration of the organoids, likely reflecting excessive cell death.

Disrupted organoids consist of dead cells and lack an intact epithelium. To investigate whether organoids with a disrupted and dark morphology in the bright-field microscope represent structures with increased cell death, we performed live/dead staining of untreated and treated organoids with Hoechst 33342 and propidium iodide (PI). Alternatively, cells were stained with calcein-acetoxymethyl ester and PI. Confocal laser scanning microscopy showed that the epithelial layer of untreated organoids was Hoechst positive, but PI negative, indicating viability of this subset of cells. Although few dead cells were observed in the epithelial layer of untreated organoids, a large number of PI-positive cells was seen in their lumen, likely representing shed and dead mature epithelial cells. Remarkably, when treated with cisplatin, the majority of the cells in the organoids became Hoechst- and PI-double positive, indicating major cell death induction. This was accompanied by a disrupted epithelial structure, as seen before in the bright-field microscopy (Figure 2a). These drastic changes upon treatment with cisplatin were confirmed using calcein staining. Although untreated organoids showed a strong calcein staining with 

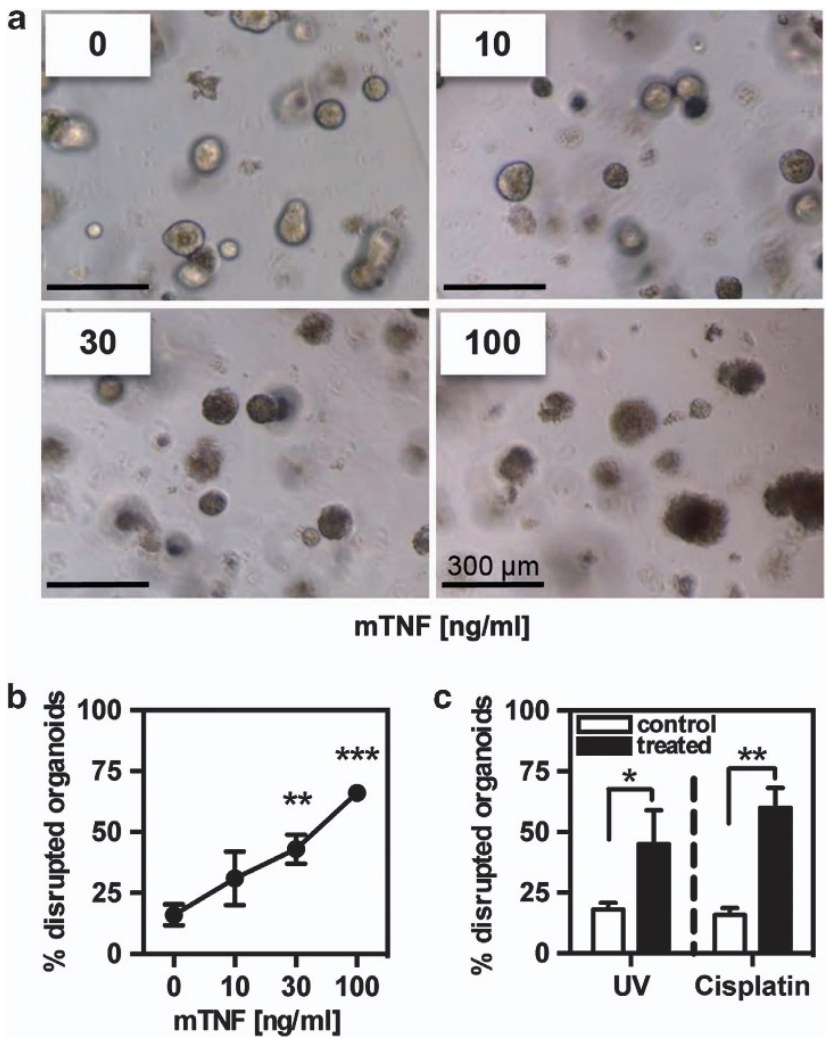

Figure 1 Changes in organoid morphology after cell death induction. Crypts from murine small intestine were seeded into Matrigel and cultured for $72 \mathrm{~h}$ to obtain organoids. (a) Organoids were treated with different doses of mTNF $\alpha(0,10,30$ and $100 \mathrm{ng} / \mathrm{ml}$ ) overnight, and morphology of the crypts was assessed by light microscopy. (b) The relative number of organoids with altered morphology was counted for each dose of TNF $\alpha$. At least 100 organoids per well were counted. Mean values \pm S.D. of triplicates are shown. Data are representative for three independent experiments. ${ }^{* *} P<0.01$ control versus $30 \mathrm{ng} / \mathrm{ml} \mathrm{TNF} \alpha$; ${ }^{* * *} P<0.001$ control versus $100 \mathrm{ng} / \mathrm{ml} T \mathrm{TNF} \alpha$. (c) Organoids were either treated overnight with cisplatin ( $10 \mu \mathrm{g} / \mathrm{ml})$ or were UV irradiated (200 $\mathrm{mJ})$, and the percentage of organoids with changed morphology was quantified using light microscopy. Mean values of three independent experiments \pm S.D. are shown. ${ }^{*} P<0.05$ control versus UV-irradiated; ${ }^{* *} P<0.01$ control versus cisplatin-treated

few PI-positive cells in the epithelial layer, cisplatin-treated organoids had reduced numbers of calcein-positive cells but increased PI-positive cells, paralleled with disrupted epithelial structures (Figure $2 b$ ). These data confirm that cell death triggers lead to organoids with a disrupted morphology, which predominantly consist of dead cells.

Viable organoids can be visualized in situ by MTT (3-(4,5-dimethylthiazol-2-yl)-2,5-diphenyltetrazolium bromide) reduction. Looking for an efficient, quantitative and objective way to measure organoid cell death, we reasoned that assessment of viability would result in more robust data, as background cell death could be subtracted. Consequently, we tried to stain the viable crypt organoids in the three-dimensional cultures using MTT. The tetrazolium salt is converted by viable cells into an insoluble purple formazan. Figure $3 a$ shows that control-treated organoids nicely converted MTT and appeared as dark purple structures in the light microscope. MTT reduction was a very rapid process in viable cells, and was readily detected already after 2 min, further increasing until $60 \mathrm{~min}$ (Figure 3d). In line with the increased number of dead cells observed with live/dead stains, a drastic reduction in formazan-positive organoids was observed after treatment with cisplatin (Figure 3a). Staining with MTT allowed simple discrimination between viable and dead intestinal organoids using bright-field microscopy. Intriguingly, the visualization of the three-dimensional structure as well as the distinction between live and dead organoids was even easier and more impressive when examined by dark-field microscopy, where formazan-positive live cells showed a red staining and dead cells had a white appearance (Figure 3b). Moreover, even a subset of dead, white cells within a viable organoid could be distinguished, which was not possible in bright-field microscopy (Figure 3b, white arrowhead). Using MTT staining, the viability of seeded organoids could also be seen on a macroscopic level. MTT-stained three-dimensional cultures of control-treated organoids showed a typical punctated pattern, which was absent upon treatment with cisplatin (Figure $3 \mathrm{c}$ ). Hence, staining with MTT provides the simplest and most rapid way to visualize viability and cytotoxicity of organoids from primary intestinal tissue.

Quantitative assessment of MTT staining in threedimensional organoid cultures. Although the distribution of MTT-positive and -negative organoids could be easily inspected by light microscopy, generation of quantitative data proved to be more cumbersome. Particularly difficult was the quantitative scoring of organoids with variable composition of live and dead cells. MTT assays are generally quantified by lysing the cells in DMSO, thereby solubilizing the formazan crystals, and measurement of absorbance at $562 \mathrm{~nm}$. As the Matrigel of the threedimensional culture does not dissolve in DMSO, an adaptation of this assay was developed. After completion of MTT staining, Matrigel was solubilized in $2 \%$ SDS, after which tetrazolium salt crystals were dissolved in DMSO and absorbance was measured at $562 \mathrm{~nm}$.

To assess whether the number of seeded crypts and the resulting viable organoids correlates with the recorded MTT absorbance, and to determine the sensitivity of this method, a serial dilution of crypts was seeded into 96-well plates. First, the linearity of theoretically seeded crypts and the number of counted organoids was determined, confirming a very high correlation (Figure 4a). Furthermore, a high correlation between visually counted organoids and the relative reduction of MTT could be demonstrated (Figure 4b). Finally, the number of theoretically seeded crypts and the relative MTT reduction was also highly correlative (Figure 4c). These data illustrate that this modified MTT assay is linear over a wide range of organoid dilutions, and correlates with the actual number of viable organoids in culture.

Next, this assay was employed to determine cell death induction in organoids after treatment with different stimuli. The viability of the organoids was normalized by resazurin metabolism before cell death treatment. Using this modified MTT assay, a concentration-dependent increase in organoid cell death in response to cisplatin and irinotecan was observed (Figure 5a). Similarly, organoids died in a 

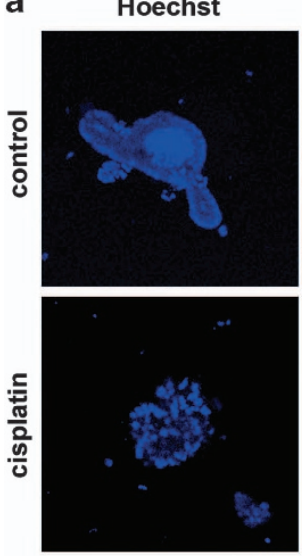

b
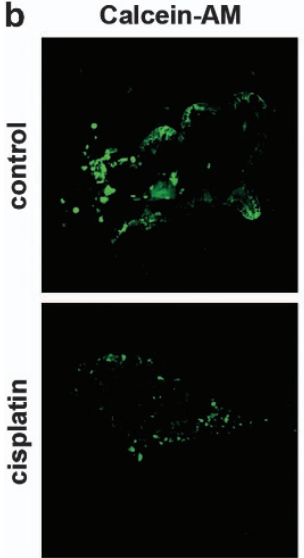

PI
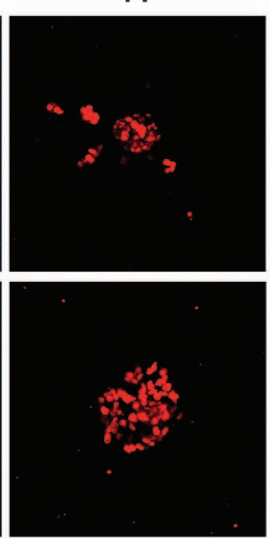

PI
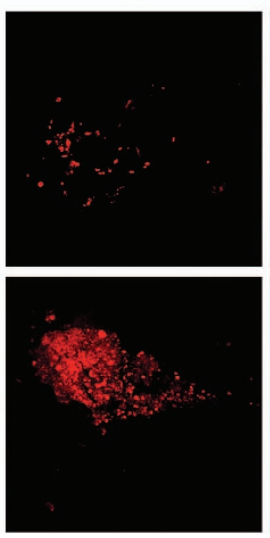

merged
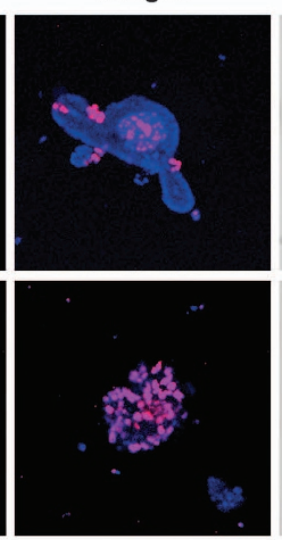

merged
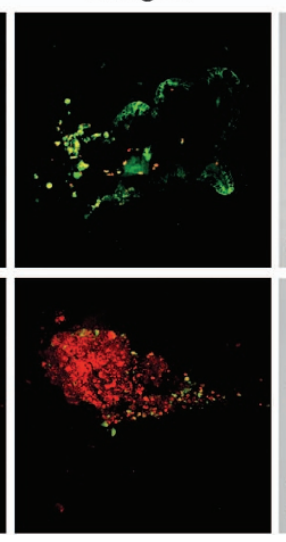

BF

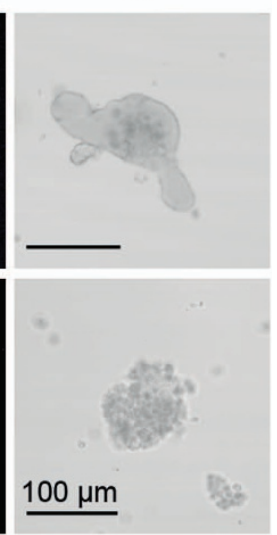

BF

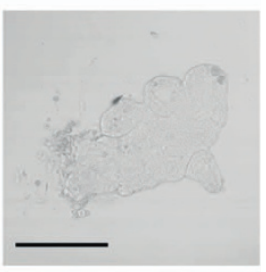

$100 \mu \mathrm{m}$

Figure 2 Live/dead staining in intestinal organoids. Intestinal crypts were isolated and cultured for $72 \mathrm{~h}$, before treatment with medium control or $30 \mu \mathrm{g} / \mathrm{ml}$ cisplatin overnight. For analysis of cell death, Matrigel was disrupted mechanically and live/dead staining solution was added. (a) $100 \mu \mathrm{g} / \mathrm{ml}$ propidium iodide (PI) and $100 \mu \mathrm{g} / \mathrm{ml}$ Hoechst 33342, or (b) $100 \mu \mathrm{g} / \mathrm{ml}$ PI plus $4 \mu \mathrm{M}$ Calcein-AM. Organoids viability was observed by confocal microscopy. BF, bright field. Data shown are representative for three independent experiments

concentration-dependent manner in response to $\mathrm{TNF} \alpha$ (Figure 5b) and X-ray irradiation (Figure 5c).

To compare these results with intestinal tumor cells, often used as surrogates for IEC, the colorectal tumor cell line Caco-2 (Figures 5d-f) and the murine intestinal carcinoma cell line MC38 (Figures 5g-i) were exposed to the same stimuli. Although primary organoids were exquisitely sensitive to cisplatin and irinotecan treatment, only reduced cell death was observed at identical concentrations in Caco-2 and MC38 cells. More drastically, TNF $\alpha$ alone failed to promote cell death in Caco-2 cells, and cells only showed a moderate response after sensitization with actinomycin D (ActD). In MC38 cells, TNF $\alpha$-induced cell death was comparable to that in primary organoids, an effect that was highly synergistic after ActD pretreatment. After X-ray irradiation, Caco-2 and MC38 cells showed to be much more resistant compared with primary organoids and only died in response to high doses. These findings demonstrate that primary intestinal organoids and colorectal tumor cells differentially respond to various cell death triggers.

Comparison of 5-fluorouracyl (5-FU)-induced cell death in vivo, ex vivo and in vitro. As ex vivo cultured intestinal organoids may represent a useful model to investigate the sensitivity of IECs to toxic substances, we aimed at directly comparing the response of intestinal crypts to the chemotherapeutic drug 5-FU in in vivo and ex vivo cultures. Mice were treated with saline control or 5-FU, and the extent of apoptosis induction was assessed $24 \mathrm{~h}$ later using cleavedcaspase 3 immunohistochemistry. Figures $6 a$ and b illustrate that while in control-treated mice minimal cell death was detected, whereas treatment of mice with 5-FU resulted in extensive apoptosis induction in intestinal crypts and mature epithelial cells. These results illustrate that the intestinal epithelium is a major target of 5-FU-induced toxicity. This strong sensitivity of intestinal crypts was confirmed in ex vivo cultured intestinal organoids, where low concentrations of 5-FU induced extensive cell death (Figure 6c). In comparison, Caco-2 and MC38 cells, used as surrogates for IECs, were found to be approximately 10-30 times less sensitive to 5-FU treatment than intestinal organoids (Figure $6 \mathrm{~d}$ and e). These findings suggest that ex vivo cultured organoids may more closely reflect the sensitivity of intestinal crypt cells in vivo.

Organoid cytotoxicity to study the impact of specific genes. Gene-deficient mice are a frequently used tool to study the role of specific genes in given physiological and pathophysiological processes. To assess whether organoids 
a

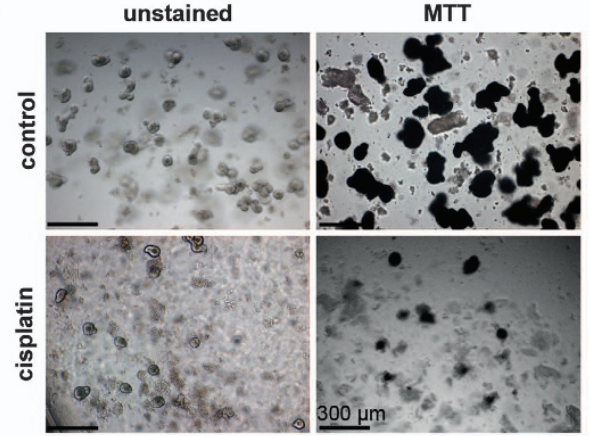

b
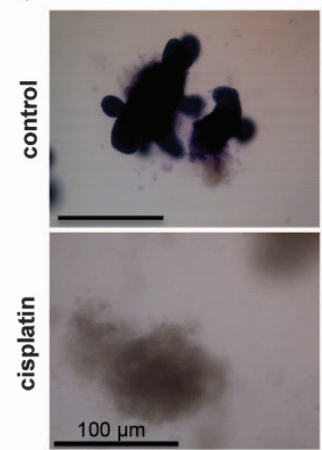

DF

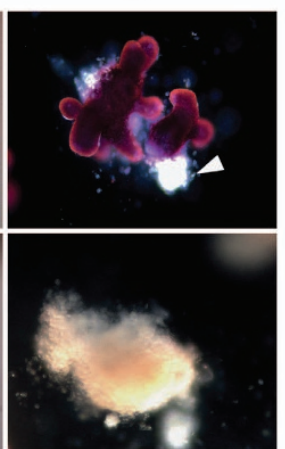

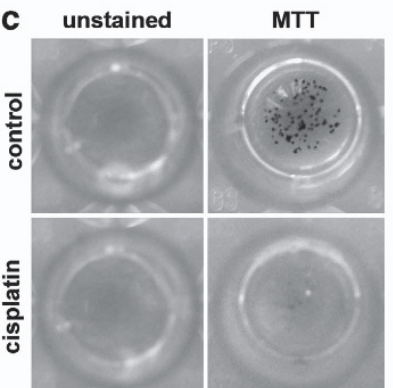

d $\quad 0$

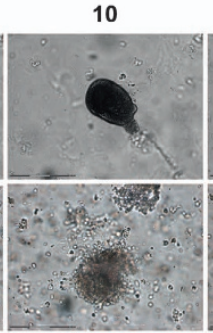

$60 \min$
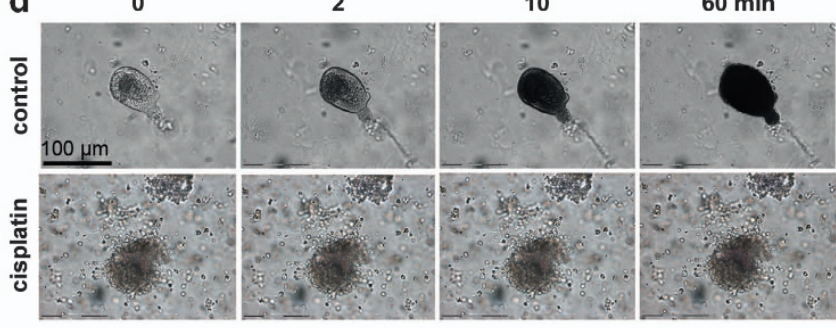

Figure 3 Visualization of organoid viability in situ using MTT reduction. Intestinal crypts were isolated and cultured in Matrigel for $72 \mathrm{~h}$. After treatment with cisplatin $(30 \mu \mathrm{g} / \mathrm{ml})$ or PBS (control) overnight, MTT solution was added to the organoids for $1 \mathrm{~h}$. (a) Left panel: unstained organoids. Right panel: MTT staining of viable organoids after control and cisplatin treatment. (b) MTT staining in single organoid after control or cisplatin treatment. Visualization of crypt viability using either bright-field (BF) or dark-field (DF) microscopy (white arrowhead: subset of dead cells). (c) Macroscopic view of unstained and MTT-stained organoids in a 96-well plate after control or cisplatin treatment. (d) Time course of MTT staining in an untreated viable versus a cisplatin-treated organoid. Data shown are representative for three independent experiments

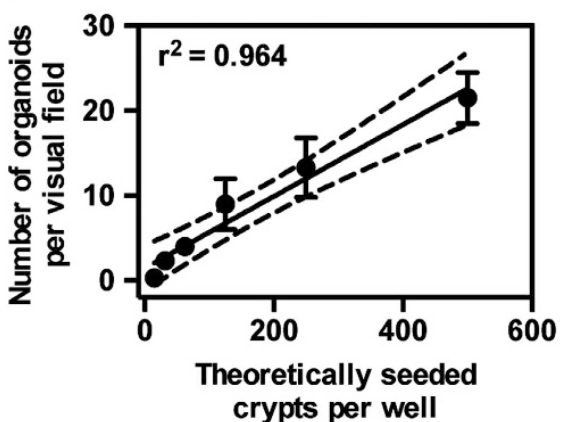

b

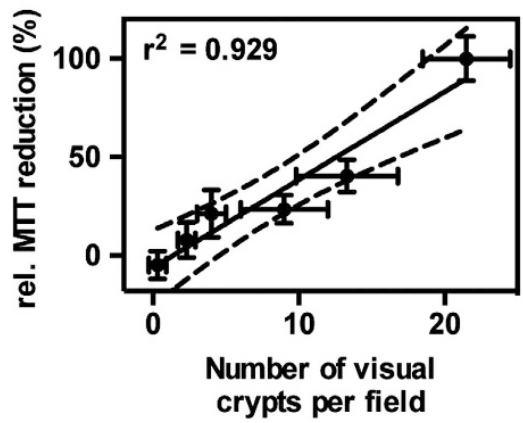

C

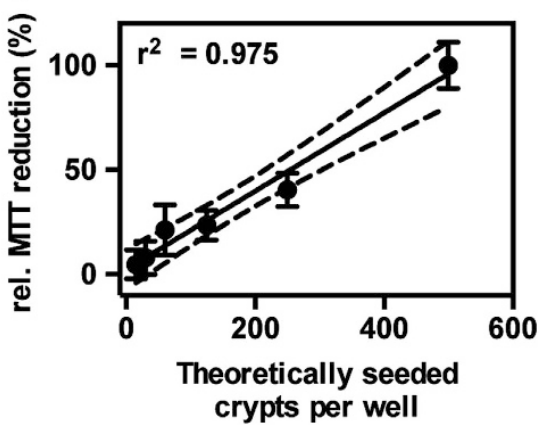

Figure 4 Quantitative measurement of organoid viability by MTT reduction. Intestinal crypts were isolated, counted and seeded in serial dilutions. Before measuring the relative MTT reduction at $562 \mathrm{~nm}$, the number of organoids per visual field (using the $\times 10$ objective) was counted by eye. MTT reduction was measured in SDS- and DMSO-solubilized organoids. (a) Correlation between theoretically seeded crypts and organoids counted per visual field. (b) Correlation between counted organoids per visual field and relative MTT reduction. (c) Correlation between theoretically seeded crypts and measured relative MTT reduction. Dotted lines indicate confidence intervals $\mathrm{Cl}_{95 \%}$ of best-fit line

could also be used to investigate the role of certain genes in the regulation of IEC death, we isolated and cultured intestinal crypts from wild-type mice and $\mathrm{Bim}^{-1-}$ mice. The pro-apoptotic $\mathrm{Bcl}-2$ homolog Bim has been implicated in a number of apoptosis signaling pathways, including responses to chemotherapeutic drugs. ${ }^{31-33}$ Coherently with the previous results, organoids from wild-type mice died after $\mathrm{TNF} \alpha$ treatment in a concentration-dependent manner, and no differences in the response was observed between wildtype and $\mathrm{Bim}^{-1-}$ organoids (Figure 7a). In marked contrast, wild-type organoids were substantially more sensitive to cisplatin treatment than $\mathrm{Bim}^{-1-}$ organoids (Figure $7 \mathrm{~b}$ ). These findings indicate that Bim is involved in cisplatininduced organoid cell death, whereas it is dispensable for TNF $\alpha$-induced apoptosis. The data further support the idea that organoids are an interesting model system to study the impact of genes on IEC death.

\section{Discussion}

Cell-based model systems are not only interesting as research tools to investigate signaling pathways, but also of 

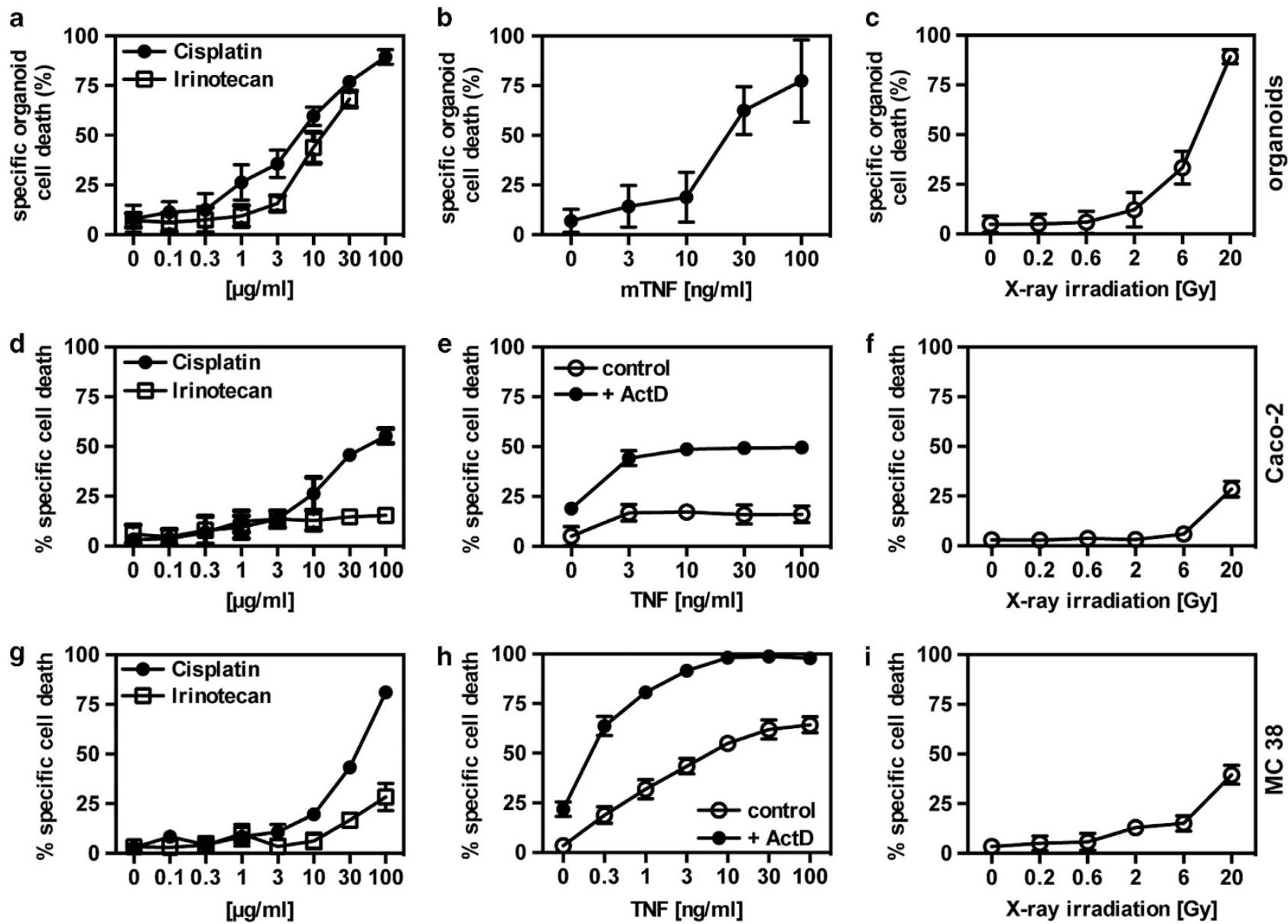

Figure 5 Quantitative measurement of organoid death in comparison to human and murine cancer cell lines. (a-c) MTT reduction assay was used for determining cell death in the organoid cultures. Crypt seeding density was normalized by resazurin reduction before treatment. Cell death was either induced by different doses of cisplatin or irinotecan (a), mTNF $\alpha$ (b) or X-ray irradiation (c). (d-f) Caco-2 cells were treated with different doses of either cisplatin or irinotecan (d), hTNF $\alpha \pm$ actinomycin D (60 ng/ml) (e), or X-ray irradiation (f) and cell death determined by MTT reduction. ( $\mathbf{g}-\mathbf{i})$ MC38 cells were treated with different doses of either cisplatin or irinotecan ( $\mathbf{g})$, $m$ TNF $\alpha \pm$ actinomycin D $(60 \mathrm{ng} / \mathrm{ml})(\mathrm{h})$ or X-ray irradiation (i) and cell death determined by MTT reduction. Each treatment was performed in triplicates. Mean values \pm S.D. are shown. Data are representative for three independent experiments with comparable results

major importance for the pharmaceutical industry to develop and test pharmaceutical drugs for their specific therapeutic effects as well as their unspecific toxicity. ${ }^{34}$ The closer these cell-based systems are to the actual target tissue, the better. Owing to their distinct gene expression profile, cells of different tissue origin markedly differ in their responses to drugs and toxins. ${ }^{35}$ Thus, therapeutic as well as adverse sideeffects should be preferentially tested in the cell type of interest. This is, however, often a major problem because of difficulties to access the tissue or to culture this cell type ex vivo. ${ }^{36}$ Although general toxic or positive therapeutic effects can be investigated in vivo using suitable animal models, these model systems often do not allow to distinguish between direct effects, or indirect and bystander effects on target tissues. Furthermore, in vivo experiments in mice and rats are often limited to final toxicity testing because of the enormous costs and ethical considerations. An alternative, in particular for in vitro investigations of biochemical processes, is represented by easy-to-culture immortalized or transformed cell lines as surrogates. However, these cell lines are by definition markedly different from the primary cells of the tissue, from which they had originally been isolated. ${ }^{37}$ The selection to survive and expand in vitro often leads to changes in gene expression profiles and altered responses. Thus, in particular for toxicological studies or investigations of cell death pathways, these cell lines are often only of very limited use as they do not represent primary cells and do not reflect the situation in vivo in the target tissue. In line with this idea, we have seen that primary organoids are exquisitely sensitive to $\mathrm{TNF} \alpha$ and chemotherapeutic drugs, reflecting the situation in vivo, whereas the colorectal tumor cell line Caco-2 showed a profound resistance to chemotherapy, and only responded moderately to TNF $\alpha$ after sensitization by ActD.

Primary cells are therefore the best model system to represent processes in the respective tissue or organ. Studies in isolated IECs have been, however, thus far particularly frustrating because of their poor survival ex vivo. In recent times, work by Clevers' group and others has led to the development of three-dimensional culture systems, which allow the culture and growth of intestinal stem cells, eventually 


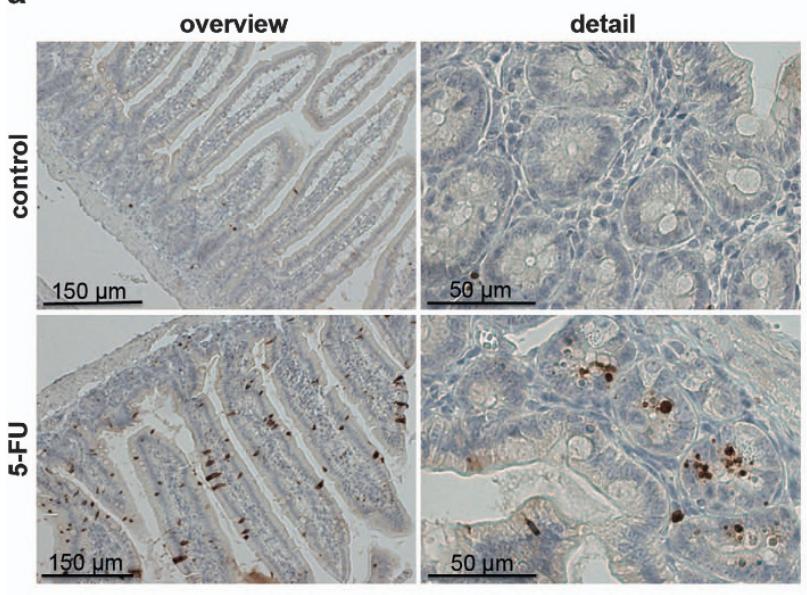

b

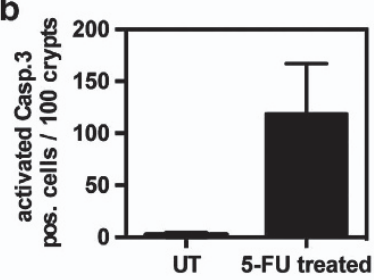

d

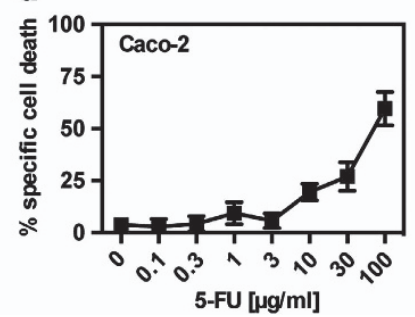

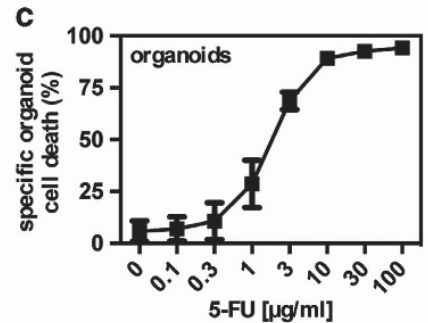

e

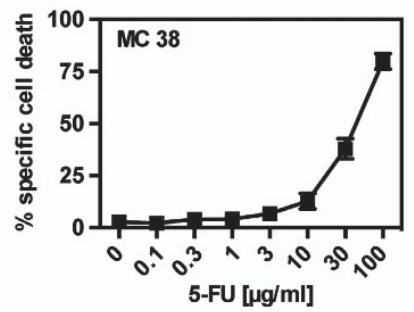

Figure 6 5-FU induced cell death in vivo, in organoids and in cancer cell lines. (a) Immunohistochemistry of small intestinal tissue sections from control or 5-FU-treated mice ( $n=3$ per group) using an anti-cleaved caspase-3 antibody. Left panel: overview; right panel: detail. (b) Organoids were cultured for $72 \mathrm{~h}$ and then treated with different concentrations of 5-FU for $24 \mathrm{~h}$. Cell death was determined by MTT reduction. Data are representative for three independent experiments. (c and d) Caco-2 cells (c) or MC 38 cells (d) were treated with different concentrations of 5-FU for $24 \mathrm{~h}$. Cell death was determined by MTT reduction. Data are representative for three independent experiments

a

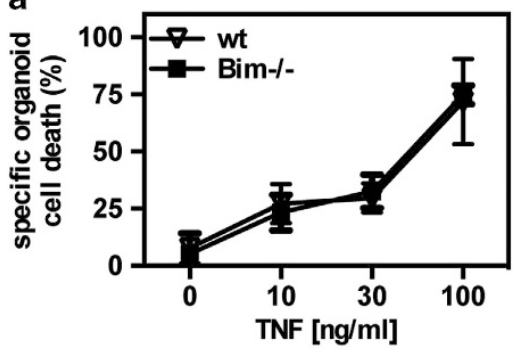

b

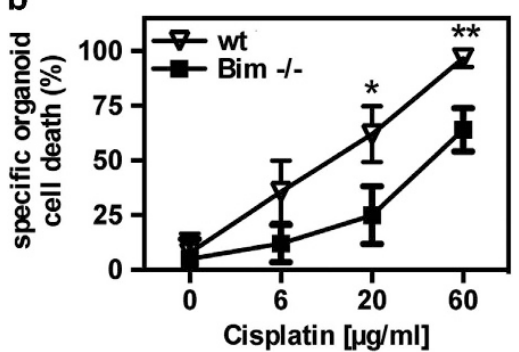

Figure 7 Role of Bim in organoid death. Crypts from wild-type (wt) or Bimdeficient $\left(\mathrm{Bim}^{-1-}\right)$ mice were isolated and cultured in Matrigel for $72 \mathrm{~h}$. Organoids were treated overnight with different concentrations of $\operatorname{TNF} \alpha(\mathbf{a})$ or cisplatin (b), and organoid death was determined by MTT assay. Mean values of three independent experiments \pm S.D. are shown. All experiments were done in triplicates. ${ }^{*} P<0.05$ wt versus $\mathrm{Bim}^{-1-} ;{ }^{* *} P<0.01$ wt versus $\mathrm{Bim}^{-1-}$

forming organoids resembling intact mature intestinal epithelial layers in their cellular composition and function. ${ }^{38}$ This ex vivo model system with primary cells has been successfully used to study stem cell biology, proliferation and differentiation of crypt cells, gene expression patterns and biochemical processes. Furthermore, the ability to culture organoids from human colorectal tumor stem cells has expanded this model system to the investigation of biological processes in primary intestinal tumor cells with great relevance for the situation in tumor patients.

Thus far, the intestinal crypt organoid model system has not been used for toxicological studies and/or investigations on cell death signaling pathways. We here demonstrate that the three-dimensional culture of intestinal crypts and their growth and differentiation to organoids is an interesting and easy to handle tool to study cell death-promoting stimuli and associated signal transduction pathways in a cellular system with great relevance for primary tissue cells. We here further describe a variety of methods to score cell death and cell survival, respectively, in these organoids using microscopical and colorimetric methods. A major problem of the microscopic methods is always the subjectivity of analysis. A large number of samples has to be analyzed and the scoring system, for example, the distinction between live and dead, has to be as objective as possible. Although theoretically cell death in organoids could be measured by a large panel of objective cell death analysis methods (e.g., Annexin V/PI staining), a critical aspect is their three-dimensional culture in Matrigel, which prevents classical flow cytometrical assays without further isolation and dissociation. Similarly, detection of DNA fragmentation by TUNEL assay or detection of cleaved caspase substrates using immunohistochemistry may be possible, but cumbersome, and not feasible for mid- and large-scale screening.

Here we now report about a critical modification of the MTT assay, which allows assessment of organoid survival using both qualitative morphological parameters as well as quantitative colorimetric measurements. Although the in situ conversion of MTT permits optical inspection using bright-field microscopy, the dissociation of the Matrigel using SDS, the solubilization in DMSO and subsequent reading in the absorbance plate reader allows to obtain quantitative and objective survival data. Our investigations also confirm that the assay correlates over a wide range of 
dilutions with the actual and microscopically inspected number of dead and live crypts or organoids in culture, respectively. Thus, this assay is accurate, reproducible and easy to handle, and nicely complements qualitative microscopy-based methods.

Three-dimensional culture of intestinal organoids may thus represent a most interesting screening system for pharmacological drugs and toxins. The possibility to dissect ex vivo cultured organoids, to freeze intestinal stem cells and to regrow them later in culture allows to develop highly reproducible test systems with defined sources of cells. Furthermore, encouraging studies on human intestinal organoids suggest that pharmacological and toxicological studies could be extended in the future to ex vivo cultured human colonic crypts, with major relevance for the potential implications of the results in human patients. ${ }^{26}$ In addition, as organoids not only consist of intestinal stem cells, but also their differentiated progeny, such as Paneth cells, Goblet cells and mature absorptive epithelial cells, toxicological studies can be performed in a model system resembling very closely the situation of the intestinal epithelial layer in vivo.

The here-described ex vivo model system not only allows the screening of pharmacological or toxic compounds, but also to study the impact of factors and triggers with high relevance for certain intestinal diseases. For example, TNF $\alpha$ has been implicated in the pathogenesis of a number of intestinal inflammatory disorders, ${ }^{39}$ and this ex vivo model system appears to largely reproduce the cell death-promoting activity of TNF $\alpha$ in vivo. ${ }^{40}$ The possibility to culture intestinal organoids in 96-well plates, and possibly even smaller formats, not only allows to screen a large number of samples and dilutions of compounds to generate quantitative data, but also represents an economical method that limits the use of expensive drugs. Last, the availability of colorectal tumor tissue as well as normal colonic epithelium from the same patient after surgery may allow in future for the side-by-side culture and testing of colorectal tumor organoids and normal colonic organoids to simultaneously test and screen drugs for their tumoricidal activity and the absence of adverse toxic side-effects on normal colonic tissue.

In summary, we here describe for the first time the use of ex vivo cultured intestinal organoids and the use of a modified MTT assay as an easy to handle and sensitive method to screen for toxic and cell death-promoting activities of compounds and treatments. This method may represent an interesting and cost-efficient alternative method to the in vivo testing in laboratory animals of drugs with an intestinal epithelium-damaging activity.

\footnotetext{
Materials and Methods

Crypt isolation, cell lines and culture conditions. Isolation and culture of intestinal crypts were done as described previously ${ }^{24}$ with modifications indicated. Briefly, the small intestine of 4- to 12-week-old C57BL/6 wild-type or Bim-deficient mice ${ }^{41}$ was opened longitudinally and villi were scraped off using a coverslip. The intestine was cut into $1-2 \mathrm{~cm}$ pieces, washed three times with cold PBS and incubated with $2 \mathrm{mM}$ EDTA in PBS for $30 \mathrm{~min}$ at $4{ }^{\circ} \mathrm{C}$ on a rotating wheel. Residual villi were removed by gentle shaking, the villi containing supernatant was removed and replaced with cold PBS. This procedure was repeated until no villi could be observed anymore in the supernatant using microscopy. Crypts were then detached from the basal membrane by vigorous shaking. The crypts enriched in the supernatant were passed through a $70-\mu \mathrm{m}$ cell
}

strainer (BD Biosciences, Heidelberg, Germany), centrifuged at $100 \times \mathrm{g}$ ( $3 \mathrm{~min}$, $4{ }^{\circ} \mathrm{C}$ ) and resuspended in $10 \mathrm{ml}$ PBS for counting using microscopy. Pelleted crypts were resuspended in Matrigel (BD Biosciences) at a desired crypt density. Two hundred to five hundred crypts in $7 \mu$ l Matrigel were seeded per well on a prewarmed 96-well flat-bottom plate and incubated for $15 \mathrm{~min}$ at $37^{\circ} \mathrm{C}$. Then, $70 \mu \mathrm{l}$ of complete crypt culture medium was added (ADF medium: advanced DMEM/F12 (Sigma, Schnelldorf, Germany), 0.1\% BSA (PAA, Cölbe, Germany), $2 \mathrm{mM}$ L-glutamine (PAA), $10 \mathrm{mM} \mathrm{HEPES} \mathrm{(Sigma),} 100 \mathrm{U} / \mathrm{ml}$ penicillin (Sigma), $100 \mu \mathrm{g} / \mathrm{ml}$ streptomycin (Sigma), $20 \mu \mathrm{g} / \mathrm{ml}$ nystatin (Sigma), $1 \mathrm{mM} \mathrm{N}$-acetyl cysteine (Sigma), $1 \times$ B27 supplement (Invitrogen, Carlsbad, CA, USA), $1 \times$ N2 supplement (Invitrogen), $50 \mathrm{ng} / \mathrm{ml} \mathrm{mEGF}$ (Peprotech, Hamburg, Germany), $100 \mathrm{ng} / \mathrm{ml} \mathrm{mNoggin}$ (Peprotech), $500 \mathrm{ng} / \mathrm{ml}$ human R-spondin-1 (Peprotech)). Alternatively, hRspondin-1 was added as conditioned medium of hR-spondin-1-transfected HEK $293 \mathrm{~T}$ cells to a final volume of $10 \%$ crypt culture medium. In some experiments, hR-spondin- 1 was omitted. Organoids were cultured at $37^{\circ} \mathrm{C}$ in a $5 \% \mathrm{CO}_{2}$ atmosphere for at least 3 days before stimulation of cell death. The human colorectal tumor cell line Caco-2 and the murine intestinal tumor cell line MC38 were obtained from ATCC (LGC, Wesel, Germany), and cultured in IMDM (Sigma) supplemented with $10 \%$ FCS (PAA), $1 \times$ MEM amino-acid solution (Sigma), 4 mM L-glutamine and $50 \mu \mathrm{g} / \mathrm{ml}$ gentamycin (Sigma) at $37^{\circ} \mathrm{C}$ and $5 \% \mathrm{CO}_{2}$.

Induction of cell death. Cell death was induced by treatment of organoids, Caco-2 cells or MC38 cells with either human TNF $\alpha$ or murine TNF $\alpha$ (Peprotech), cisplatin or irinotecan (CPT11, Enzo Life Sciences, Lörrach, Germany), 5-FU (Teva, Harleem, the Netherlands), UV irradiation (Stratalinker, Stratagene, La Jolla, CA, USA) or X-ray irradiation (using a X-Rad 225 ix source from Precision $X$-Ray Inc., Branford, CT, USA). In some experiments, Caco-2 or MC38 cells were sensitized to TNF $\alpha$-mediated cell death by pre-treatment with $60 \mathrm{ng} / \mathrm{ml} \mathrm{ActD}$ (Sigma) $1 \mathrm{~h}$ before treatment with TNF $\alpha$.

Determination of organoid cell death by counting. Organoids were counted before treatment at the budding stage after 2-3 days of culture. A minimum of 100 crypts were counted and defined as viable or dead by their morphological appearance in the bright-field microscope (Axiovert 25, Zeiss, Oberkochen, Germany). After that, organoids were treated overnight with the indicated cell death-inducing agents in triplicates. Finally, the organoids were counted and scored as viable or dead. The specific toxicity of the respective trigger was calculated as $\%$ viable $_{\text {after }}-\%$ viable $_{\text {before }}$.

Assessment of crypt viability via MTT reduction. After 3-4 days of culture, organoids were exposed overnight to different cell death inducers, and organoid viability was assessed via the ability to reduce MTT. When indicated, viability of seeded organoids was normalized before apoptosis induction by incubation with resazurin. Briefly, organoids were cultured with $10 \mu \mathrm{g} / \mathrm{ml}$ resazurin (Sigma) for $6 \mathrm{~h}$, the supernatant was transferred into a black 96-well plate and fluorescence was measured with a fluorescence detector (Wallac, Victor 1420 multilabel counter, Perkin-Elmer, Rodgau, Germany) at $530 \mathrm{~nm}$ excitation and $590 \mathrm{~nm}$ emission. After overnight treatment with death-inducing stimuli, MTT (Sigma) solution was added to the organoid culture to a final concentration of $500 \mu \mathrm{g} / \mathrm{ml}$. After incubation for $2-3 \mathrm{~h}$ at $37^{\circ} \mathrm{C}, 5 \% \mathrm{CO}_{2}$, the medium was discarded and $20 \mu \mathrm{l}$ of $2 \%$ SDS (Sigma) solution in $\mathrm{H}_{2} \mathrm{O}$ was added to solubilize the Matrigel $\left(2 \mathrm{~h}, 37^{\circ} \mathrm{C}\right)$. Then, $100 \mu \mathrm{l}$ of DMSO were added for $1 \mathrm{~h}\left(37^{\circ} \mathrm{C}\right)$ to solubilize the reduced MTT, and the $\mathrm{OD}$ was measured on a microplate absorbance reader (Sunrise Tecan Reader, Männedorf, Germany) at $562 \mathrm{~nm}$. Seven microliters of Matrigel without organoids were used as control and set as $0 \%$ viability. Untreated organoids were defined as $100 \%$ viable. If normalization with resazurin was done before treatment, the relative MTT reduction was divided by the fluorescence value of respective wells.

Fluorescence confocal microscopy. Cell death in organoids was assessed by live/dead staining and subsequent fluorescence confocal microscopy. Therefore, Matrigel was disrupted mechanically with a pipette tip and organoids were transferred to an Eppendorf tube. Crypts were stained in ADF medium with either $100 \mu \mathrm{g} / \mathrm{ml}$ Hoechst 33342 (Sigma) and $100 \mu \mathrm{g} / \mathrm{ml} \mathrm{PI} \mathrm{(Sigma),} \mathrm{or} 4 \mu \mathrm{M}$ calcein-acetoxymethyl ester (Invitrogen) and $100 \mu \mathrm{g} / \mathrm{ml} \mathrm{PI}$. After $30 \mathrm{~min}$ of incubation in the dark, confocal microscopy was performed on a laser scanning microscope (LSM 510 META, Zeiss).

Microscopy. For microscopic analysis, single untreated or treated organoids were isolated from the Matrigel under the microscope using a micropipette. 
The organoids were placed onto a glass slide and covered with a coverslip. Brightfield, phase-contrast and dark-field microscopy were done on a Leitz DMRB (Leica, Solms, Germany) or an Axiovert 25 (Zeiss) microscope. The density of organoids per visual field was obtained by counting using the $\times 10$ objective. Microscopy of treated or untreated organoids within the Matrigel was done on a PALM MicroBeam microscope (Zeiss).

In vivo induction of apoptosis and immunohistochemistry. 5 -FU-induced apoptosis in IECs was done as part of a different study. EGFP-IRESCreERT2xAPCfl/fl mice under C57BI/6 background were treated with tamoxifen (Sigma) to induce Cre recombinase. Three weeks later, mice were treated with PBS control or with $30 \mathrm{mg} / \mathrm{kg} 5$-FU. After $24 \mathrm{~h}$, mice were killed, and small intestinal tissue samples were formalin fixed and paraffin embedded. Apoptotic cell death in tissue sections was then detected using an anti-cleaved caspase 3 antibody (Asp175, Cell Signaling Technology, Danvers, MA, USA) and protocols used were as described previously. ${ }^{42}$ All the sections were observed and photographed with the PALM MicroBeam microscope (Zeiss). Cleaved caspase 3 positive cells per 100 crypts were scored in adenoma-free areas of the small intestine. Three mice per group were analyzed.

Statistics. All statistical analyses were performed using the Graph Pad Prism software (Ver. 5.01) (GraphPad Software, San Diego, CA, USA). For correlation analysis, linear regression was used to obtain the best-fit line. Also the $95 \%$ confidence interval of the best-fit line and the coefficient of determination $\left(r^{2}\right)$ were calculated and shown for linear regression. All experiments were performed in triplicates, if not stated otherwise. All values are reported as mean \pm S.D. Where indicated, statistical analysis was evaluated with unpaired Student's $t$-tests. A $P$ value of $<0.05$ was considered statistically significant. For independent experiments, organoids were isolated from different individual C57BL/6 mice.

\section{Conflict of Interest}

The authors declare no conflict of interest.

Acknowledgements. We thank the Brunner laboratory for constructive discussion and technical help. This work was supported by an Equipment Grant from the German Research Foundation (DFG) (INST 38/500-1) to T Brunner. $T$ Grabinger was supported by a fellowship from the DFG-supported Research Training Group RTG 1331

1. Clevers H. The intestinal crypt, a prototype stem cell compartment. Cell 2013; 154 274-284.

2. Bullen TF, Forrest S, Campbell F, Dodson AR, Hershman MJ, Pritchard DM et al Characterization of epithelial cell shedding from human small intestine. Lab Invest 2006; 86: $1052-1063$

3. Vereecke L, Beyaert R, van Loo G. Enterocyte death and intestinal barrier maintenance in homeostasis and disease. Trends Mol Med 2011; 17: 584-593.

4. Sanders DS. Mucosal integrity and barrier function in the pathogenesis of early lesions in Crohn's disease. J Clin Pathol 2005; 58: 568-572.

5. Salim SY, Soderholm JD. Importance of disrupted intestinal barrier in inflammatory bowel diseases. Inflamm Bowel Dis 2011; 17: 362-381.

6. Maiuri L, Ciacci C, Raia V, Vacca L, Ricciardelli I, Raimondi F et al. FAS engagement drives apoptosis of enterocytes of coeliac patients. Gut 2001; 48: 418-424.

7. Washington K, Jagasia M. Pathology of graft-versus-host disease in the gastrointestinal tract. Hum Pathol 2009; 40: 909-917.

8. Duprez L, Takahashi N, Van Hauwermeiren F, Vandendriessche B, Goossens V, Vanden Berghe $T$ et al. RIP kinase-dependent necrosis drives lethal systemic inflammatory response syndrome. Immunity 2011; 35: 908-918.

9. Suzuki T. Regulation of intestinal epithelial permeability by tight junctions. Cell Mol Life Sci 2013; 70: 631-659.

10. Kaser A, Niederreiter L, Blumberg RS. Genetically determined epithelial dysfunction and its consequences for microflora-host interactions. Cell Mol Life Sci 2011; 68: 3643-3649.

11. Marini M, Bamias G, Rivera-Nieves J, Moskaluk CA, Hoang SB, Ross WG et al. TNF-alpha neutralization ameliorates the severity of murine Crohn's-like ileitis by abrogation of intestinal epithelial cell apoptosis. Proc Natl Acad Sci USA 2003; 100 : 8366-8371.

12. Piguet PF, Vesin C, Guo J, Donati Y, Barazzone C. TNF-induced enterocyte apoptosis in mice is mediated by the TNF receptor 1 and does not require p53. Eur J Immunol 1998; 28: 3499-3505.
13. Lin T, Brunner T, Tietz B, Madsen J, Bonfoco E, Reaves $\mathrm{M}$ et al. Fas ligand- mediated killing by intestinal intraepithelial lymphocytes. Participation in intestinal graft-versus-host disease. J Clin Invest 1998; 101: 570-577.

14. Juuti-Uusitalo K, Klunder LJ, Sjollema KA, Mackovicova K, Ohgaki R, Hoekstra D et al. Differential effects of TNF (TNFSF2) and IFN-gamma on intestinal epithelial cell morphogenesis and barrier function in three-dimensional culture. PLoS One 2011; 6: e22967.

15. Kalive M, Zhang W, Chen Y, Capco DG. Human intestinal epithelial cells exhibit a cellular response indicating a potential toxicity upon exposure to hematite nanoparticles. Cell Biol Toxicol 2012; 28: 343-368

16. Sergent JA, Paget V, Chevillard S. Toxicity and genotoxicity of nano-SiO2 on human epithelial intestinal HT-29 cell line. Ann Occup Hyg 2012; 56: 622-630.

17. Sun $\mathrm{C}$, Fang $\mathrm{H}$, Xie T, Auth RD, Patel N, Murray PR et al. Anthrax lethal toxin disrupts intestinal barrier function and causes systemic infections with enteric bacteria. PLOS One 2012; 7 : e33583.

18. Valverde I, Lago J, Vieites JM, Cabado AG. In vitro approaches to evaluate palytoxin-induced toxicity and cell death in intestinal cells. J Appl Toxicol 2008; 28: 294-302.

19. Xian CJ. Roles of growth factors in chemotherapy-induced intestinal mucosal damage repair. Curr Pharm Biotechnol 2003; 4: 260-269.

20. Yeoh AS, Gibson RJ, Yeoh EE, Bowen JM, Stringer AM, Giam KA et al. A novel animal model to investigate fractionated radiotherapy-induced alimentary mucositis: the role of apoptosis, p53, nuclear factor-kappaB, COX-1, and COX-2. Mol Cancer Ther 2007; 6 : 2319-2327.

21. Bowen JM, Gibson RJ, Stringer AM, Chan TW, Prabowo AS, Cummins AG et al. Role of p53 in irinotecan-induced intestinal cell death and mucosal damage. Anticancer Drugs 2007; 18: 197-210.

22. Grossmann J, Maxson JM, Whitacre CM, Orosz DE, Berger NA, Fiocchi C et al. New isolation technique to study apoptosis in human intestinal epithelial cells. Am J Pathol 1998; 153: $53-62$

23. Kutuk $O$, Letai A. Alteration of the mitochondrial apoptotic pathway is key to acquired paclitaxel resistance and can be reversed by ABT-737. Cancer Res 2008; 68: 7985-7994.

24. Sato T, Vries RG, Snippert HJ, van de Wetering M, Barker N, Stange DE et al. Single Lgr5 stem cells build crypt-villus structures in vitro without a mesenchymal niche. Nature 2009; 459: 262-265.

25. Gunther C, Martini E, Wittkopf N, Amann K, Weigmann B, Neumann H et al. Caspase-8 regulates TNF-alpha-induced epithelial necroptosis and terminal ileitis. Nature 2011; 477 : 335-339.

26. Sato $T$, Stange DE, Ferrante M, Vries RG, Van Es JH, Van den Brink S et al. Long-term expansion of epithelial organoids from human colon, adenoma, adenocarcinoma, and Barrett's epithelium. Gastroenterology 2011; 141: 1762-1772.

27. Sala FG, Kunisaki SM, Ochoa ER, Vacanti J, Grikscheit TC. Tissue-engineered small intestine and stomach form from autologous tissue in a preclinical large animal model. J Surg Res 2009; 156: 205-212.

28. Kuratnik A, Giardina C. Intestinal organoids as tissue surrogates for toxicological and pharmacological studies. Biochem Pharmacol 2013; 85: 1721-1726.

29. Stelzner M, Helmrath M, Dunn JC, Henning SJ, Houchen CW, Kuo C et al. A nomenclature for intestinal in vitro cultures. Am J Physiol Gastrointest Liver Physiol 2012; 302: G1359-G1363.

30. Goretsky T, Dirisina R, Sinh P, Mittal N, Managlia E, Williams DB et al. p53 mediates TNF-induced epithelial cell apoptosis in IBD. Am J Pathol 2012; 181 . $1306-1315$

31. Corazza N, Jakob S, Schaer C, Frese S, Keogh A, Stroka D et al. TRAIL receptor-mediated JNK activation and Bim phosphorylation critically regulate Fas-mediated liver damage and lethality. J Clin Invest 2006; 116: 2493-2499.

32. Schmich K, Schlatter R, Corazza N, Sa Ferreira K, Ederer M, Brunner T et al. Tumor necrosis factor alpha sensitizes primary murine hepatocytes to Fas/CD95induced apoptosis in a Bim- and Bid-dependent manner. Hepatology 2011; 53: 282-292.

33. Sidler D, Brockmann A, Mueller J, Nachbur U, Corazza N, Renzulli $P$ et al. Thiazolide-induced apoptosis in colorectal cancer cells is mediated via the Jun kinaseBim axis and reveals glutathione-S-transferase P1 as Achilles' heel. Oncogene 2012; 31: 4095-4106.

34. Bouvier d'Yvoire M, Bremer S, Casati S, Ceridono M, Coecke S, Corvi R et al. ECVAM and new technologies for toxicity testing. Adv Exp Med Biol 2012; 745: 154-180.

35. Svoboda M, Riha J, Wlcek K, Jaeger W, Thalhammer T. Organic anion transporting polypeptides (OATPs): regulation of expression and function. Curr Drug Metab 2011; 12 : 139-153.

36. Allen DD, Caviedes R, Cardenas AM, Shimahara T, Segura-Aguilar J, Caviedes PA. Cell lines as in vitro models for drug screening and toxicity studies. Drug Dev Ind Pharm 2005; 31: $757-768$

37. Muscella A, Vetrugno C, Fanizzi FP, Manca C, De Pascali SA, Marsigliante S. A new platinum(II) compound anticancer drug candidate with selective cytotoxicity for breast cancer cells. Cell Death Dis 2013; 4: e796. 
38. Sato $\mathrm{T}$, Clevers $\mathrm{H}$. Growing self-organizing mini-guts from a single intestinal stem cell: mechanism and applications. Science 2013; 340: 1190-1194.

39. Silva LC, Ortigosa LC, Benard G. Anti-TNF-alpha agents in the treatment of immune-mediated inflammatory diseases: mechanisms of action and pitfalls. Immunotherapy 2010; 2: 817-833.

40. Lau KS, Juchheim AM, Cavaliere KR, Philips SR, Lauffenburger DA, Haigis KM. In vivo systems analysis identifies spatial and temporal aspects of the modulation of TNF-alphainduced apoptosis and proliferation by MAPKs. Sci Signal 2011; 4: ra16.

41. Bouillet P, Metcalf D, Huang DC, Tarlinton DM, Kay TW, Kontgen F et al. Proapoptotic $\mathrm{Bcl}-2$ relative Bim required for certain apoptotic responses, leukocyte homeostasis, and to preclude autoimmunity. Science 1999; 286: 1735-1738

42. Jakob S, Corazza N, Diamantis E, Kappeler A, Brunner T. Detection of apoptosis in vivo using antibodies against caspase-induced neo-epitopes. Methods 2008; 44: 255-261.
Cell Death and Disease is an open-access journal published by Nature Publishing Group. This work is licensed under a Creative Commons Attribution-NonCommercialShareAlike 3.0 Unported License. The images or other third party material in this article are included in the article's Creative Commons license, unless indicated otherwise in the credit line; if the material is not included under the Creative Commons license, users will need to obtain permission from the license holder to reproduce the material. To view a copy of this license, visit http://creativecommons.org/licenses/ by-nc-sa/3.0/

Supplementary Information accompanies this paper on Cell Death and Disease website (http://www.nature.com/cddis) 